\title{
Regular Positive-Real Functions and Passive Networks Comprising Two Reactive Elements
}

\author{
Jason Zheng Jiang and Malcolm C. Smith
}

\begin{abstract}
The purpose of this paper is to formalise the concept of a regular positive-real function and to demonstrate its usefulness in the classification of low-complexity two-terminal networks. We will develop a series of lemmas characterising the basic properties of regularity. We will give a simple proof that all five-element series-parallel networks with two reactive elements are regular, and we will show that there are only two bridge-networks with two reactive elements which may be non-regular. We will discuss the class of two-terminal networks with two reactive elements and an arbitrary number of resistive elements and highlight a theorem of Reichert. The results are motivated by an approach to passive mechanical control which makes use of the inerter device.
\end{abstract}

\section{INTRODUCTION}

A famous theorem in electrical networks by Bott and Duffin [1] showed that any positive-real function could be realised as the driving-point immittance of a network consisting of resistors, capacitors and inductors only. The apparent non-minimality of the construction has subsequently intrigued many researchers and there were a number of important papers which followed up on this question, e.g. [2], [3]. Interest in the topic lost momentum in the early 1970s due to the growing importance of integrated circuits. Recently, a new network element (the inerter) was introduced for mechanical control [4] which has revived interest in passive network realisations. The inerter is a mechanical twoterminal element with the property that the applied force at the terminals is proportional to the relative acceleration across the terminals. Applications of the method to vehicle suspension [5], [6], control of motorcycle steering instabilities [7], [8] and vibration absorption [4] have been identified. The inerter has been successfully deployed in Formula One racing since 2005 [9].

For mechanical realisations, minimising network complexity is important. As such, a systematic classification of the realisability conditions of simple networks is needed. Within the electrical circuit literature there was an attempt by a number of authors in the 1960s and early 1970s to carry out such a classification [10], [11], [12], [13], [14], [15], [16]. Many partial results were established but a complete picture was never obtained, even for the apparently simple case of a biquadratic. Today, this literature is not easy to master, in part because there is no convincing summary of the results that were obtained. This paper represents a fresh look at this topic and represents an attempt to rework, simplify and generalise existing results and provide an accessible entry point into this field.

Both authors are with the Department of Engineering, University of Cambridge, Cambridge CB2 1PZ, U.K.; zj219@cam.ac.uk, mcs@eng.cam.ac.uk
The present paper formalises the concept of a regular positive-real function and demonstrates its usefulness in the classification of low-complexity two-terminal networks. A series of lemmas will be developed to aid in this classification. This paper also introduces the terminology of a network quartet which consists of four networks related by frequency inversion and duality. It will be seen that the class of regular biquadratic functions can be realised with two network quartets and that many other networks fall within the same class in a simple manner. Among the five-element networks with two reactive elements it will be shown that there is only one network quartet which contains two bridge networks that can realise non-regular immittances.

The conditions for a biquadratic positive-real function to be regular can be found in the paper of Vasiliu [15]. However, the present paper goes further in formalising this concept and exploring its properties in a systematic manner. The concept illuminates results of Foster, Ladenheim and Lin and connects to the important theorem of Reichert [14]. The concept of a network quartet has appeared in the German literature with the terminology "Untergruppe" [17].

\section{The Concept of Regularity And Its Properties}

Definition: A positive-real function $Z(s)$ is defined to be regular if the smallest value of $\operatorname{Re}(Z(j w))$ or $\operatorname{Re}\left(Z^{-1}(j w)\right)$ occurs at $\omega=0$ or $w=\infty$.

Lemma 1: Let $Z(s)$ be a regular positive-real function. Then $\alpha Z(s), Z\left(\beta^{-1} s\right), Z\left(s^{-1}\right), Z^{-1}(s)$ are all regular, where $\alpha, \beta>0$.

Proof: Obvious.

Lemma 2: Let $Z(s)$ be a regular positive-real function. Then $Z(s)+R$ and $Z^{-1}(s)+R^{-1}$ are both regular, where $R>0$.

Proof: Consider $Z(s)+R$. If $\operatorname{Re}(Z(j w))$ achieves its minimum when $w=0$ or $w=\infty$, the conclusion is trivial. Let us assume that $\operatorname{Re}\left(Z^{-1}(j w)\right)$ achieve its minimum at $w=0$. Let $Z^{-1}(j w)=Y_{R}(j w)+j Y_{I}(j w)$, where $Y_{R}, Y_{I}$ are real functions. By assumption, $Y_{R}(0) \leq Y_{R}(j w)$ for all $w$ and $Y_{I}(0)=0$. Then

$$
\begin{aligned}
& \operatorname{Re}\left((Z(j w)+R)^{-1}\right) \\
= & \frac{R^{-1}\left(Y_{R}(j w)\left(Y_{R}(j w)+R^{-1}\right)+Y_{I}(j w)^{2}\right)}{\left(Y_{R}(j w)+R^{-1}\right)^{2}+Y_{I}(j w)^{2}} \\
\geq & \frac{R^{-1} Y_{R}(j w)}{Y_{R}(j w)+R^{-1}} \\
\geq & \frac{R^{-1} Y_{R}(0)}{Y_{R}(0)+R^{-1}}=\operatorname{Re}\left((Z(0)+R)^{-1}\right) .
\end{aligned}
$$


So $Z(s)+R$ is regular. A similar argument holds if $\operatorname{Re}\left(Z^{-1}(j w)\right)$ achieves its minimum at $w=\infty$. The case of $Z^{-1}(s)+R^{-1}$ is similar.

Lemmas 1 and 2 imply that if a network can only realise regular immittances, then so will the dual network (if it exists), the network obtained by exchanging the inductors (springs) and capacitors (inerters) and the network obtained by adding a resistor (damper) in series or in parallel with the original one.

Lemma 3: The positive-real driving point impedance $Z(s)$ and admittance $Y(s)$ of any network that has all reactive elements of the same kind satisfies $\operatorname{Re}(Z(j w))$ and $\operatorname{Re}(Y(j w))$ being monotonic ([18, Chapter 2.2]) and hence can only realise regular immittances.

Lemma 4: Any network that has a path $\left(1,1^{\prime}\right)$ or cut set $\left(1,1^{\prime}\right)$ consisting of one type of reactive elements can only realise regular immittances. ( 1 and $1^{\prime}$ are the two external terminals of the network; path $\left(1,1^{\prime}\right)$ refers to a path between these terminals, and cut set $\left(1,1^{\prime}\right)$ is a cut set which places 1 and $1^{\prime}$ in different connected parts.)

Proof: This follows since either the impedance or the admittance has a zero at $s=0$ or $\infty$.

We now focus attention on biquadratic positive-real functions

$$
Z(s)=\frac{A s^{2}+B s+C}{D s^{2}+E s+F},
$$

where $A, B, C, D, E, F \geq 0$. It can be shown that $Z(s)$ is positive real if and only if

$$
\sigma=B E-(\sqrt{A F}-\sqrt{C D})^{2} \geq 0 .
$$

Lemma 5: A positive-real biquadratic impedance (1) is regular if and only if at least one of the following four conditions is satisfied:

$$
\begin{aligned}
\text { Case 1. } & A F-C D \geq 0 \text { and } \\
& \lambda_{1}=E(B F-C E)-F(A F-C D) \geq 0,
\end{aligned}
$$

Case 2. $A F-C D \geq 0$ and

$$
\lambda_{2}=B(A \bar{E}-B D)-A(A F-C D) \geq 0,
$$

Case 3. $A F-C D \leq 0$ and

$$
\lambda_{3}=D(A \bar{F}-C D)-E(A E-B D) \geq 0,
$$

Case 4. $A F-C D \leq 0$ and

$$
\lambda_{4}=C(A \bar{F}-C D)-B(B F-C E) \geq 0 .
$$

Proof: Without loss of generality, let us assume $\operatorname{Re}(Z(j w))$ achieves its minimum when $w=0$. This is equivalent to $Z(s)-Z(0)$ being positive-real, which happens if and only if the conditions of Case 1 are satisfied. Similar arguments hold for the other three cases.

Lemma 6: A positive-real biquadratic impedance (1) with $Z(0)=Z(\infty) \neq 0, \infty$ (which implies $A F-C D=0$ ) is regular.

Proof: It can be seem from Lemma 5 that when $A F-$ $C D=0$, at least two of the cases must be satisfied.

Lemma 7: A positive-real biquadratic impedance (1) with the resultant $K=(A F-C D)^{2}-(A E-B D)(B F-C E) \leq$ 0 can be realised by at most one kind of reactive element, hence is regular.
Proof: If $K=0$, the numerator and denominator have a factor in common, and so the function would reduce immediately to no more than a bilinear function, which is regular by our definition. Foster [10], [11] points out that if $K$ is negative, the reactive elements are of the same kind. Based on Lemma 3, impedance (1) must be regular.

Lemma 8: A positive-real biquadratic impedance (1) with any of the parameters $A, B, C, D, E$ and $F$ equals zero is regular. Moreover, any such impedance can be realised by a series-parallel network with at most two reactive elements and two resistive elements.

Proof: If $B$ or $E$ equals zero, since (2) holds, $A F=$ $C D$. Based on Lemma 6, (1) is always regular. If any of $A$, $C, D, F$ equals zero, this follows since either the impedance or the admittance has a zero at $s=0$ or $\infty$. In any of the above cases the Foster preamble will result in a network of the stated form.

\section{A CANONICAL FORM FOR BIQUADRATICS AND NETWORK QUARTETS}

The classification of networks is facilitated by the following transformations on the impedance $Z(s)$ :

1) Extraction of a constant multiplier $\alpha$,

2) Frequency scaling: $s \rightarrow \beta^{-1} s$,

3) Frequency inversion: $s \rightarrow s^{-1}$,

4) Impedance inversion: $Z \rightarrow Z^{-1}$.

In network realisations, the first two transformations correspond to element scaling, the third to an interchange of inductors (springs) and capacitors (inerters), and the fourth to taking the network dual. We remark that a network has a dual if and only if it is planar. Furthermore, a two-terminal network with seven branches or less is guaranteed to be planar [19].

Based on Lemma 8, without loss of generality, we can assume that $A, B, C, D, E, F>0$ for (1). Using the first two transformations, $Z(s)$ can be reduced to a canonical form

$$
Z_{c}(s)=\frac{s^{2}+2 U \sqrt{W} s+W}{s^{2}+(2 V / \sqrt{W}) s+1 / W},(U, V, W>0)
$$

where $\alpha=A / D$ and $\beta=\sqrt[4]{A D /(C F)}$. It is easily verified that $W=C \beta^{2} / A=D \beta^{-2} / F, U=B \beta /(2 A \sqrt{W})$, $V=E \beta \sqrt{W} /(2 D)$. Clearly, any circuit that realises $Z_{c}(s)$ will also realise $Z(s)$ after the appropriate transformation of the elements $(R \rightarrow \alpha R, L \rightarrow \beta L / \alpha, C \rightarrow \alpha C / \beta)$. Thus, for the realisation of the biquadratic, it is sufficient to restrict attention to the class in (3). This canonical form was first considered in [14].

Next we observe that frequency inversion $\left(s \rightarrow s^{-1}\right)$ corresponds to the transformation $W \rightarrow W^{-1}$ in the canonical form and impedance inversion (duality) corresponds to the transformations: $U \rightarrow V, W \rightarrow W^{-1}$. This means that, if the realisability conditions in terms of $U, V, W$ are known for a given network, they can be written down routinely for a quartet of related networks. This is illustrated in Fig. 1 where 


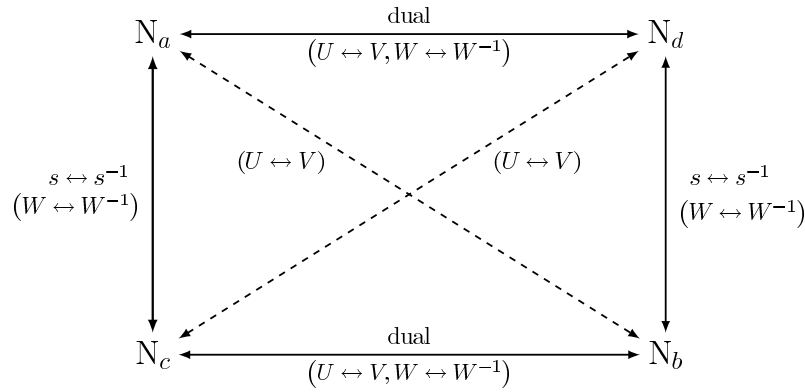

Fig. 1. Transformations relating members of a network quartet.

the four networks $\mathrm{N}_{a}, \mathrm{~N}_{b}, \mathrm{~N}_{c}, \mathrm{~N}_{d}$ are related by interchange of inductors (springs) and capacitors (inerters) (denoted by $s \leftrightarrow s^{-1}$ ) and duality, and the corresponding transformations of $U, V, W$ in the canonical form.

From the above it is clear that the set of $U, V, W$ which can be realised by a quartet of networks is invariant under both transformations: $U \leftrightarrow V$ and $W \leftrightarrow W^{-1}$. It is obvious from Lemma 1 that the transformations 1) - 4) do not change the regularity of a given impedance, and if one of the networks in a network quartet can only realise regular immittances, so will the other networks in this quartet.

It follows from (2) that $Z_{c}(s)$ is positive real if and only if

$$
\sigma_{c}=4 U V+2-\left(\frac{1}{W}+W\right) \geq 0 .
$$

The resultant of $Z_{c}(s)$ is

$$
K_{c}=4 U^{2}+4 V^{2}-4 U V\left(\frac{1}{W}+W\right)+\left(\frac{1}{W}-W\right)^{2} .
$$

For any polynomial $\rho(U, V, W)$ we introduce the notation $\rho^{*}(U, V, W)=\rho\left(U, V, W^{-1}\right)$. We can now restate Lemmas 5-7 for the canonical form (3):

Lemma 9: Let $Z_{c}(s)$ be a positive-real biquadratic defined in (3). Then:

1) $Z_{c}(s)$ is regular if and only if at least one of the following four conditions is satisfied:

Case $1 . W \leq 1$ and

$$
\epsilon_{1}=4 U V-4 V^{2} W-\left(\frac{1}{W}-W\right) \geq 0,
$$

Case 2. $W \leq 1$ and

$$
\epsilon_{2}=4 U V-4 U^{2} W-\left(\frac{1}{W}-W\right) \geq 0,
$$

Case 3. $W \geq 1$ and $\epsilon_{1}^{*} \geq 0$,

Case 4. $W \geq 1$ and $\epsilon_{2}^{*} \geq 0$.

2) When $W=1, Z_{c}(s)$ is regular.

3) When $K_{c} \leq 0, Z_{c}(s)$ can be realised by at most one kind of reactive element, hence is regular.

We now illustrate the regions in the $(U, V)$-plane characterised in Lemma 9 which correspond to a regular biquadratic for $W \leq 1$. Clearly, when $(U, V)$ is in the shaded region of Fig. 2, (3) is not positive-real, hence cannot be realised passively. When $(U, V)$ is in the hatched region, (3) is regular. The remaining white region in the $(U, V)$-plane contains all the non-regular positive real impedances of (3). It follows from Lemma 3 that the region of $K_{c}<0$ is always constrained in the intersection of the region $\epsilon_{1}>0$ and

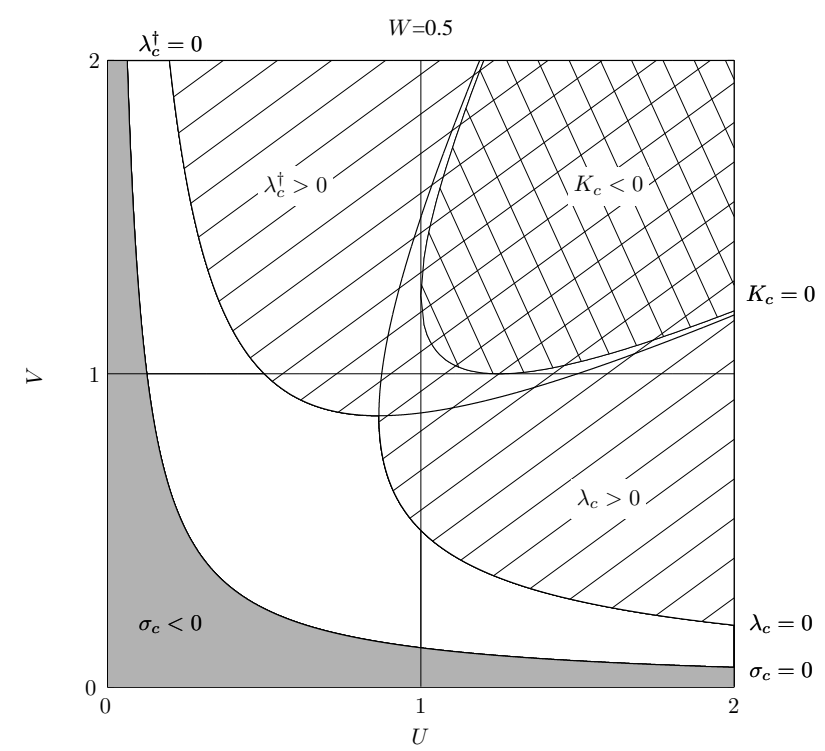

Fig. 2. The regular impedances of (3) with $W \in(0,1)$.

$\epsilon_{2}>0$. We call the region with $\epsilon_{1} \geq 0$ or $\epsilon_{2} \geq 0$ the regular region with $W<1$. This plot can also be found in [14]. For the case $W>1$, we obtain a plot which is visually identical with curves generated by $\epsilon_{1}^{*}$ and $\epsilon_{2}^{*}$ instead of $\epsilon_{1}$ and $\epsilon_{2}$. The regular region with $W>1$ is defined by $\epsilon_{1}^{*} \geq 0$ or $\epsilon_{2}^{*} \geq 0$.

\section{Five-Element Two-Reactive Element SYNTHESIS OF THE BIQUADRATIC}

\section{A. Series-Parallel Networks}

Lemma 10: The network quartet in Fig. 3 (which contains only two distinct networks) with all elements positive and finite can only realise regular immittances.

Proof: The impedance of the network shown in Fig. 3(a) can be calculated to be

$$
Z(s)=\frac{L_{1} C_{1} R_{2} s^{2}+\left(R_{1} R_{2} C_{1}+L_{1}\right) s+R_{1}}{C_{1} L_{1} s^{2}+C_{1}\left(R_{1}+R_{2}\right) s+1}
$$

Comparing (1) with (4) we obtain $k A=L_{1} C_{1} R_{2}, k B=$ $R_{1} R_{2} C_{1}+L_{1}, k C=R_{1}, k D=C_{1} L_{1}, k E=C_{1}\left(R_{1}+R_{2}\right)$ and $k F=1$, where $k$ is any positive constant. Substituting $A, B, C, D, E$ and $F$ we obtain the following expressions corresponding to Cases 1-4 in Lemma 5:

$$
\begin{aligned}
k^{2}(A F-C D) & =C_{1} L_{1}\left(R_{2}-R_{1}\right) \\
k^{3} \lambda_{1} & =C_{1} R_{1}\left(2 L_{1}-C_{1} R_{1}\left(R_{1}+R_{2}\right)\right), \\
k^{3} \lambda_{2} & =C_{1} L_{1}\left(C_{1}^{2} R_{1} R_{2}^{3}-L_{1}^{2}\right) \\
k^{3} \lambda_{3} & =C_{1}^{2} L_{1} R_{2}\left(2 L_{1}-C_{1} R_{2}\left(R_{1}+R_{2}\right)\right), \\
k^{3} \lambda_{4} & =C_{1}^{2} R_{1}^{3} R_{2}-L_{1}^{2} .
\end{aligned}
$$




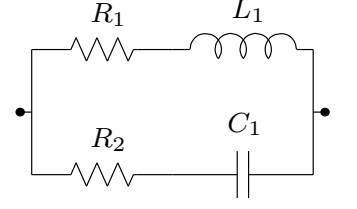

(a)

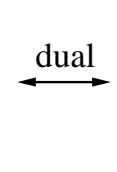

(b)

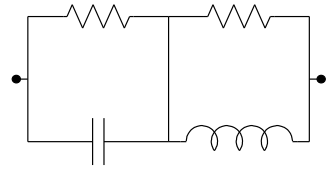

Fig. 3. The network realisation of the impedance in Lemma 10 and the other network in this quartet.

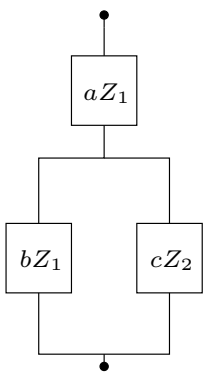

(a)

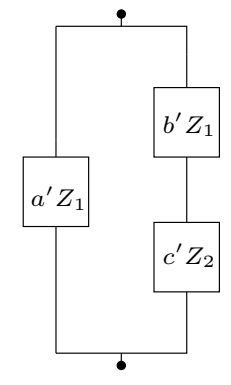

(b)
Fig. 4. Two equivalent networks.

Let us assume that $R_{2} \geq R_{1}$, which means that $A F-C D$ is nonnegative. Suppose $\lambda_{2} \leq 0$, so $L_{1} \geq C_{1} R_{2} \sqrt{R_{1} R_{2}}$. Then

$$
\begin{aligned}
& 2 L_{1}-C_{1} R_{1}\left(R_{1}+R_{2}\right) \\
\geq & 2 C_{1} R_{2} \sqrt{R_{1} R_{2}}-C_{1} R_{1}\left(R_{1}+R_{2}\right) \\
\geq & 2 C_{1}\left(R_{2} \sqrt{R_{1} R_{2}}-R_{1} R_{2}\right) \\
\geq & 0 .
\end{aligned}
$$

So $\lambda_{1}$ is nonnegative. Hence when $R_{2} \geq R_{1}, \lambda_{1}$ and $\lambda_{2}$ cannot both be negative. The case that $R_{2} \leq R_{1}$ is similar in that $\lambda_{3}$ and $\lambda_{4}$ cannot both be negative. Hence the impedance (4) must satisfy the conditions of one of the four cases in Lemma 5.

Note that the network of Fig. 3(b) is the dual network of the network shown in Fig. 3(a). Based on Lemma 1, the network of Fig. 3(b) can only realise regular immittances.

Lemma 11: ([20]) For arbitrary impedances $Z_{1}(s), Z_{2}(s)$ and positive constants $a, b, c$, the networks of Fig. 4(a) and (b) are equivalent under the transformations: $a^{\prime}=a+b$, $b^{\prime}=a(a+b) / b, c^{\prime}=c((a+b) / b)^{2}\left[a=a^{\prime} b^{\prime} /\left(a^{\prime}+b^{\prime}\right)\right.$, $\left.b=a^{\prime 2} /\left(a^{\prime}+b^{\prime}\right), c=c^{\prime}\left(a^{\prime} /\left(a^{\prime}+b^{\prime}\right)\right)^{2}\right]$.

Theorem 1: A biquadratic impedance (3) can be realised by series-parallel five-element networks with two reactive elements if and only if it is regular. Moreover, all regular biquadratics (3) can be realised by the network quartet of Fig. $5\left(K_{c}<0\right)$ or Fig. $6\left(K_{c}>0\right)$. Furthermore, only two networks from the quartet of Fig. 5 are needed to cover all cases when $K_{c}<0$ (Table I).

Proof: Sufficiency. In case $K_{c}=0$, (3) is bilinear or a constant and can be realised with at most two resistors and one reactive element. In case $K_{c}<0$, the poles and zeros of (3) lie on the negative real axis and interlace each other [18, Chapter 2.2]. There are various standard forms of networks that realise these cases, e.g. the network quartet of (a)
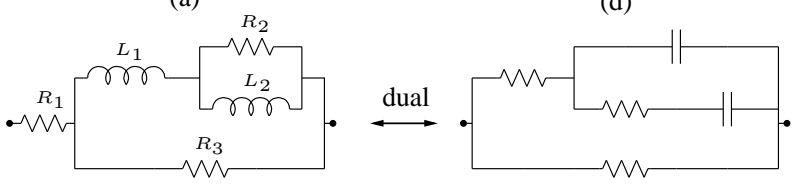

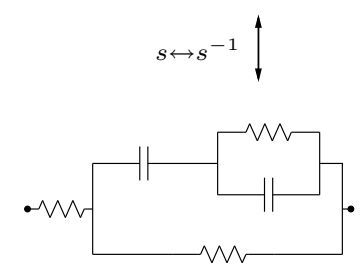

(c)

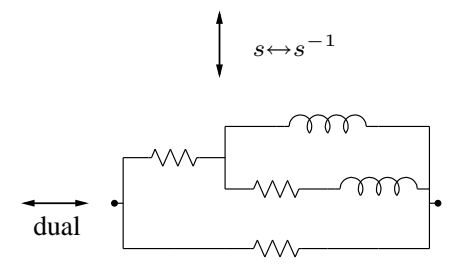

(b)
Fig. 5. The series-parallel five-element network quartet with two reactive elements that can realise all the regular biquadratic impedances (3) with $K_{c}<0$. (a)

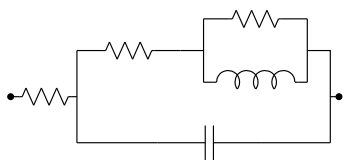

(c)

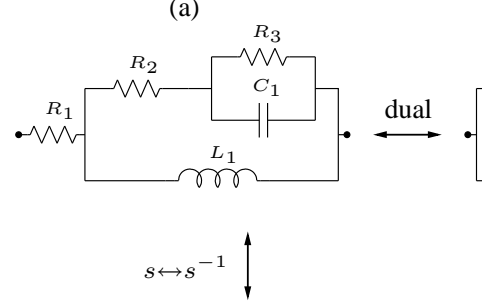

(d)
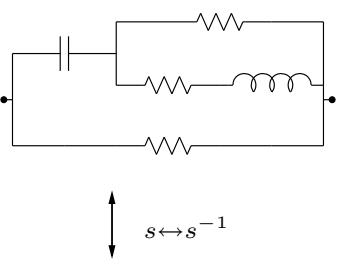

(b)

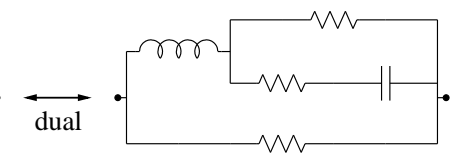

Fig. 6. The series-parallel five-element network quartet with two reactive elements that can realise all the regular biquadratic impedances (3) with $K_{c}>0$.

Fig. 5, according to the conditions of Table I. For $K_{c}>0$, the "Foster preamble" [21], [22] corresponding to each of the four cases in Lemma 9-1) leads to the realisations as the network quartet of Fig. 6 according to the conditions of Table I. It can be checked that when $K_{c}<0, W$ cannot equal 1 ; when $K_{c}>0$, if $W=1$ or any of $\epsilon_{1,2}$ and $\epsilon_{1,2}^{*}$ are zero the realisation may have fewer than five elements.

Necessity. If no distinction is made among the elements, there are 24 distinct two-terminal series-parallel structures with five elements [23]. These structures may be divided into two classes, any structure in one class having its dual in the other. Based on Lemma 1, the analysis may be performed upon only one class. In Fig. 7, all the series-parallel fiveelement structures in one class are presented. Based on Lemma 3 , we only need to investigate the networks with two reactive elements of different kinds. Based on Lemmas 2 and 4, structures $1-5,8,10$ and 11 are eliminated. Using in addition Lemma 10, structures 6, 7 and 9 are eliminated. The final elimination of structure 12 uses Lemma 11 as well. 
TABLE I

THE REALIS ABILITY CONDITIONS OF THE NETWORK QUARTETS SHOWN IN FIG. 5 AND 6 FOR THE BIQUADRATIC IMPEDANCE (3).

\begin{tabular}{|l|l|}
\hline Networks & Realisability Conditions \\
\hline Fig. 5(a), (b) & $K_{c}<0, W<1$ \\
\hline Fig. 5(c), (d) & $K_{c}<0, W>1$ \\
\hline Fig. 6(a) & $K_{c}>0, W<1, \epsilon_{1}>0$ \\
\hline Fig. 6(b) & $K_{c}>0, W<1, \epsilon_{2}>0$ \\
\hline Fig. 6(c) & $K_{c}>0, W>1, \epsilon_{1}^{*}>0$ \\
\hline Fig. 6(d) & $K_{c}>0, W>1, \epsilon_{2}^{*}>0$ \\
\hline
\end{tabular}

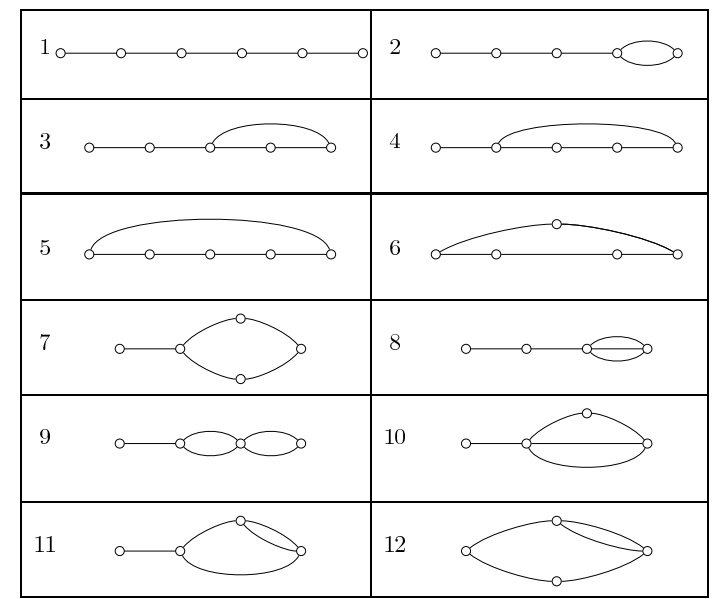

Fig. 7. One-Half of the Five-Element Series-Parallel Structures.

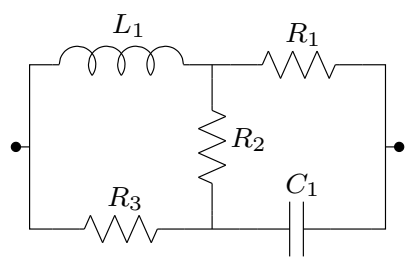

Fig. 8. The two-reactive five-element bridge network quartet which based on Lemma 12 can only realise regular impedances (1).

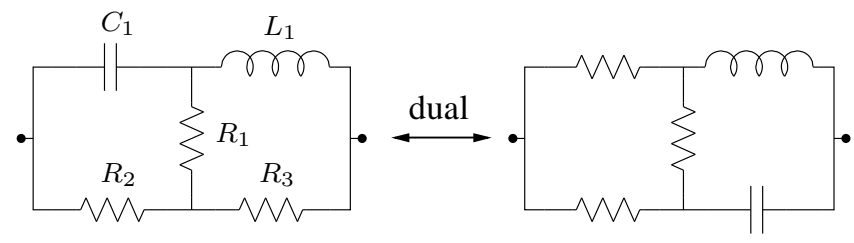

Fig. 9. The two-reactive five-element bridge network quartet that can transfer to series-parallel networks by $Y \rightarrow \Delta$ and $\Delta \rightarrow Y$ transformations.

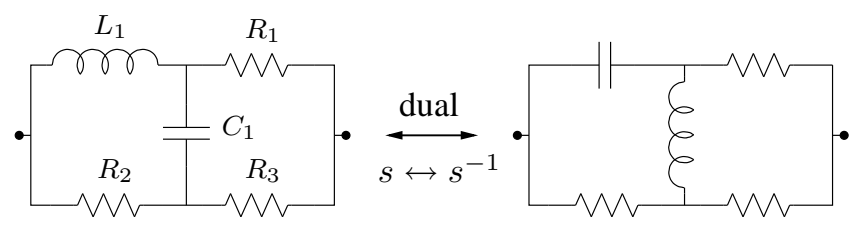

(a)

(b)

Fig. 10. The two-reactive five-element bridge network quartet that can realise non-regular impedances (1).

\section{B. Bridge Networks}

Lemma 12: The network shown in Fig. 8 with all elements positive and finite can only realise regular immittances.

Proof: See [24].

Theorem 2: All bridge networks with two reactive and three resistive elements can only realise regular immittances except for the network quartet of Fig. 10.

Proof: See [24].

We can verify that the network of Fig. 10(a) can realise the impedance function

$$
Z(s)=\frac{11 s^{2}+17 s+12}{3 s^{2}+4 s+7}
$$

with $R_{1}=2, R_{2}=2, R_{3}=10, L_{1}=2$ and $C_{1}=1 / 4$. It can be checked that (5) does not belong to any of the four cases in Lemma 5, and hence fails to be regular. Based on Lemma 1, the network of Fig. 10(b) can realise nonregular impedances as well. The necessary and sufficient realisability conditions and the non-regular realisable regions for the network quartet of Fig. 10 can be found in [24].

\section{Minimum Reactive Realis ations of the BIQUADRATIC WITH AN ARBITRARY NUMBER OF RESISTIVE ELEMENTS}

The following result of Lin [20] can be given a new proof using the methods of this paper.

Theorem 3: Any series-parallel one-port network consisting of two reactive elements and an arbitrary number of resistive elements is equivalent to a network consisting of two reactive elements and no more than three resistive elements.

Proof: Consider a finite collection of elements consisting of an arbitrary (finite) number of resistors (dampers) plus two reactive elements. We will consider a sequence of steps where at each stage two existing elements or two-terminal networks are connected together in series or in parallel to obtain a new two-terminal network. Using this procedure, any series-parallel network which can be formed from the original set of elements can be obtained. After each step we will carry out any obvious simplifications of the new network to obtain a simpler but equivalent two-terminal network. For example, if two resistors (dampers) are connected in series or in parallel they will be reduced again to a single resistor (damper).

Now suppose that at some stage the two reactive elements belong to different networks. Then we claim that the collection of networks consists of isolated resistors (dampers), or networks comprising one reactive element and at most two resistors (dampers) in either of the equivalent forms of Fig. 4. This situation will only change when the two networks containing a reactive element are combined together at some step. In this step, a network with two reactive elements and at most four resistors (dampers) will be obtained. It is easily seen that the number of resistors in this network can always be reduced to three and that the resulting network has a regular immittance. Any subsequent step involves a series or parallel connection of this network with a resistor (damper), which by Lemma 2 , does not change the immittance of 


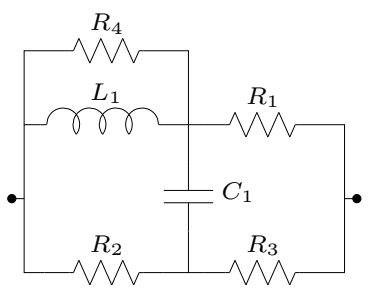

(a)

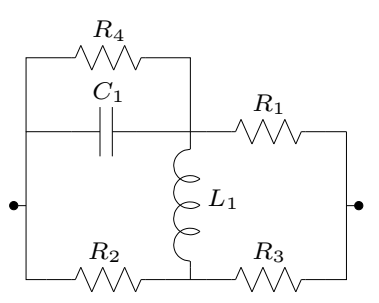

(b)
Fig. 11. The two networks in [25, Fig. (6)] that can realise non-regular biquadratics.

the network from being regular. Hence, by Theorem 1, any series-parallel network with two reactive elements is equivalent to one which uses at most three resistive elements.

It has been shown in [25] that four resistors are sufficient to synthesize the biquadratic functions with one inductor and one capacitor. Using the concept of regularity we can deduce here a stronger result.

Theorem 4: A biquadratic admittance $Y(s)$ can be realised with at most two reactive elements if and only if $Y(s)$ satisfies one of the following conditions:

1) $Y(s)$ is regular,

2) $Y(s)$ is the driving-point admittance of the networks shown in Fig. 11(a) or Fig. 11(b).

Proof: In [25], it is shown that the relevant class of mechanical admittances can be parametrised in terms of five circuit arrangements each containing four resistors (dampers). Based on Lemma 2, Lemma 12 and Theorem 1, three of the networks can only realise regular admittances. The remaining two networks are shown in Fig. 11(a) and (b). It can be seen that they are slight generalisations of the networks of Figs. 10(a) and (b).

In the terminology of the present paper we express the main result of [14] as follows.

Theorem 5: (Reichert [14]) A biquadratic admittance $Y(s)$ can be realised with at most two reactive elements if and only if $Y(s)$ satisfies one of the following conditions:

1) $Y(s)$ is regular,

2) $Y(s)$ is the driving-point admittance of the networks shown in Fig. 10(a) or Fig. 10(b).

Both Theorem 4 and Theorem 5 make use of a general necessary and sufficient condition of paramountcy to characterise transformerless resistive 3-port [26]. Evidently, the non-regular positive-real functions which can be realised using one inductor and one capacitor are covered by the bridge networks of Fig. 10(a), (b) or Fig. 11(a), (b).

\section{CONCLUSIONS}

This paper has introduced the formal concept of regularity for positive-real functions. A number of lemmas were presented illustrating the basic properties. The concept was shown to be useful in classifying the passive networks comprising two reactive elements. This set was seen to be covered by six networks which realise all regular impedances plus two bridge networks.

\section{REFERENCES}

[1] R. Bott and R. J. Duffin, "Impedance synthesis without use of transformers," J. Appl. Phys., vol. 20, p. 816, 1949.

[2] R. H. Pantell, "A new method of driving point impedance synthesis," Proc. IRE, vol. 42, p. 861, 1954.

[3] S. Seshu, "Minimal realizations of the biquadratic minimum function," IRE Transactions on Circuit Theory, pp. 345-350, Dec. 1959.

[4] M. C. Smith, "Synthesis of mechanical networks: the inerter," IEEE Trans. Automatic Control, vol. 47, no. 10, pp. 1648-1662, 2002.

[5] C. Papageorgiou and M. C. Smith, "Positive real synthesis using matrix inequalities for mechanical networks: application to vehicle suspension," IEEE Trans. on Contr. Syst. Tech., vol. 14, pp. 423-435, 2006.

[6] M. C. Smith and F.-C. Wang, "Performance benefits in passive vehicle suspensions employing inerters," Vehicle System Dynamics, vol. 42, pp. 235-237, 2004.

[7] S. Evangelou, D. J. Limebeer, R. S. Sharp, and M. C. Smith, "Control of motorcycle steering instabilities - passive mechanical compensators incorporating inerters," IEEE Control Systems Magazine, pp. 78-88, October 2006.

[8] _ , "Mechanical steering compensation for high-performance motorcycles," Transactions of ASME, J. of Applied Mechanics, vol. 74, pp. 332-346, 2007.

[9] Cambridge University Engineering Department news item, 19 August 2008. [Online]. Available: http://www.eng.cam.ac.uk/news/stories/ 2008/McLaren/

[10] R. M. Foster, "Academic and theoretical aspects of circuit theory," in Proc. IRE, vol. 50, May 1962, pp. 866-871.

[11] — - "A class of biquadratic impedances," IEEE Trans. on Circuit Theory, pp. 262-265, 1963.

[12] E. L. Ladenheim, "A special biquadratic structure," IEEE Trans. on Circuit Theory, pp. 455-456, 1963.

[13] —, "Three-reactive five-element biquadratic structures," IEEE Trans. on Circuit Theory, pp. 88-97, 1964.

[14] M. Reichert, "Die kanonisch und übertragerfrei realisierbaren Zweipolfunktionen zweiten Grades (transformerless and canonic realisation of biquadratic immittance functions)," Arch. Elek. Übertragung, vol. 23, pp. 201-208, Apr. 1969.

[15] C. G. Vasiliu, "Series-parallel six-element synthesis of the biquadratic impedances," IEEE Trans. on Circuit Theory, pp. 115-121, 1970.

[16] - "Correction to 'series-parallel six-element synthesis of the biquadratic impedances',” p. 207, November 1970.

[17] G. Dittmer, "Zur realisierung von RLC-Brückenzweipolen mit zwei Reaktanzen und mehr als drei Widerständen (on the realisation of RLC two-terminal bridge networks with two reactive and more than three resistive elements)," Nachrichtentechnische Zeitschrift, vol. 23, pp. 225-230, 1970.

[18] E. A. Guillemin, Synthesis of Passive Networks. John Wiley \& Sons, 1957.

[19] S. Seshu and M. B. Reed, Linear Graphs and Electrical Networks. Addison-Wesley, 1961.

[20] P. M. Lin, "A theorem on equivalent one-port networks," IEEE Trans. on Circuit Theory, vol. CT-12, pp. 619-621, Dec. 1965.

[21] M. E. V. Valkenburg, Introduction to Modern Network Synthesis. John Wiley \& Sons, 1960.

[22] J. E. Storer, Passive Network Synthesis. McGraw-Hill Book Company, 1957.

[23] J. Riordan and C. E. Shannon, "The number of two-terminal seriesparallel networks," J. Math. Phys., vol. 21, 1942.

[24] J. Z. Jiang and M. C. Smith, "Regular positive-real functions and five-element network realisations for passive mechanical control," in preparation.

[25] M. Z. Q. Chen and M. C. Smith, "Electrical and mechanical passive network synthesis,", in Recent Advances in Learning and Control. Springer-Verlag, 2008.

[26] B. D. H. Tellegen, "Theorie der wisselstromen," P. Noordhoff, 1952. 